\title{
KAMENEV TYPE THEOREMS FOR SECOND ORDER MATRIX DIFFERENTIAL SYSTEMS
}

\author{
LYNN H. ERBE, QINGKAI KONG, AND SHIGUI RUAN
}

(Communicated by Charles Pugh)

\begin{abstract}
We consider the second order matrix differential systems (1) $\left(P(t) Y^{\prime}\right)^{\prime}+Q(t) Y=0$ and (2) $Y^{\prime \prime}+Q(t) Y=0$ where $Y, P$, and $Q$ are $n \times n$ real continuous matrix functions with $P(t), Q(t)$ symmetric and $P(t)$ positive definite for $t \in\left[t_{0}, \infty\right) \quad\left(P(t)>0, t \geq t_{0}\right)$. We establish sufficient conditions in order that all prepared solutions $Y(t)$ of (1) and (2) are oscillatory. The results obtained can be regarded as generalizing well-known results of Kamenev in the scalar case.
\end{abstract}

\section{INTRODUCTION}

Consider the second order linear differential system

$$
\left(P(t) Y^{\prime}\right)^{\prime}+Q(t) Y=0
$$

where $t \geq t_{0}$ and $Y(t), P(t)$, and $Q(t)$ are $n \times n$ real continuous matrix functions with $P(t), Q(t)$ symmetric and $P(t)$ positive definite for $t \in\left[t_{0}, \infty\right)$ $\left(P(t)>0, t \geq t_{0}\right)$. When $P(t) \equiv I$ for $t \geq t_{0}$ where $I$ is the $n \times n$ identity matrix, we consider

$$
Y^{\prime \prime}+Q(t) Y=0 .
$$

A solution $Y(t)$ of (1.1) (or (1.2)) is said to be nontrivial if $\operatorname{det} Y(t) \neq 0$ for at least one $t \in\left[t_{0}, \infty\right)$ and a nontrivial solution $Y(t)$ of $(1.1)$ is said to be prepared if

$$
Y^{*}(t) P(t) Y^{\prime}(t)-Y^{* \prime}(t) P(t) Y(t) \equiv 0, \quad t \in\left[t_{0}, \infty\right),
$$

where for any matrix $A$, the transpose of $A$ is denoted by $A^{*}$. System (1.1) is said to be oscillatory on $\left[t_{0}, \infty\right)$ in case the determinant of every nontrivial prepared solution vanishes on $[T, \infty)$ for each $T>t_{0}$.

For the corresponding scalar equation of system (1.2),

$$
y^{\prime \prime}+q(t) y=0,
$$

Received by the editors June 13, 1991.

1991 Mathematics Subject Classification. Primary 34A30, 34C10.

Key words and phrases. Matrix differential system, oscillation theory, Riccati equation. 
the most important simple oscillation criterion is the well-known Fite-Wintner theorem which states that if $q(t) \in C\left[t_{0}, \infty\right)$ and satisfies

$$
\lim _{t \rightarrow \infty} \int_{t_{0}}^{t} q(s) d s=\infty,
$$

then equation (1.4) is oscillatory. In fact Fite [8] assumed in addition that $q(t)$ is nonnegative, while Wintner [19] proved a stronger result which required a weaker condition involving the integral average, i.e.,

$$
\lim _{T \rightarrow \infty} \frac{1}{T} \int_{t_{0}}^{T} \int_{t_{0}}^{t} q(s) d s d t=\infty .
$$

In a different direction, Hartman [9] showed that (1.4) is oscillatory in case

$$
-\infty<\liminf _{T \rightarrow \infty} \frac{1}{T} \int_{t_{0}}^{T} \int_{t_{0}}^{t} q(s) d s d t<\limsup _{T \rightarrow \infty} \frac{1}{T} \int_{t_{0}}^{T} \int_{t_{0}}^{t} q(s) d s d t .
$$

Another type of criterion was given by Kamenev [12] who showed that if for some positive integer $m>2$,

$$
\limsup _{t \rightarrow \infty} \frac{1}{t^{m-1}} \int_{t_{0}}^{t}(t-s)^{m-1} q(s) d s=\infty
$$

then equation (1.4) is oscillatory.

For matrix systems (1.1) and (1.2), many authors, cf. Allegretto and Erbe [1], Etgen and Lewis [7], Hartman [10], Hinton and Lewis [11], Tomastik [17] and Walters [18], etc., have obtained that (1.1) (or (1.2)) is oscillatory if a corresponding scalar equation obtained by applying a positive linear functional is oscillatory. Other recent oscillation criteria for (1.1) and (1.2) have involved conditions on the eigenvalues of $P(t)$ and $Q(t)$ or their integrals. if

It was conjectured by Hinton and Lewis [11] that equation (1.2) is oscillatory

$$
\lim _{t \rightarrow \infty} \lambda_{1}\left[\int_{t_{0}}^{t} Q(s) d s\right]=\infty
$$

where $\lambda_{1}(A) \geq \lambda_{2}(A) \geq \cdots \geq \lambda_{n}(A)$ denotes the usual ordering of the eigenvalues of the symmetric matrix $A$. This conjecture was settled with additional assumptions on the rate of growth of the trace of $\int_{t_{0}}^{t} Q(s) d s$ by Mingarelli [15], Kwong et al. [14], and Butler and Erbe [3, 4]. The conjecture was finally settled in the case $n=2$ by Kwong and Kaper [13] and for arbitrary $n$ by Byers, Harris, and Kwong [6].

Recently, Butler, Erbe, and Mingarelli [5] gave additional criteria for oscillation of (1.2) based on Riccati techniques and variational principles. These criteria extended the scalar criteria (1.6) and (1.7). In this paper, using Riccati techniques we establish oscillation criteria for system (1.1) and extend the Kamenev type criterion (1.8) to the matrix equation (1.2).

\section{MAIN RESUlts}

Theorem 1. Let $H(t, s)$ and $h(t, s)$ be continuous on $D=\left\{(t, s): t \geq s \geq t_{0}\right\}$ such that $H(t, t)=0$ for $t \geq t_{0}$ and $H(t, s)>0$ for $t>s \geq t_{0}$. We 
assume further that the partial derivative $\frac{\partial H}{\partial s}(t, s) \equiv H_{s}(t, s)$ is nonpositive and is continuous for $t \geq s \geq t_{0}$, and $h(t, s)$ is defined by

$$
H_{s}(t, s)=-h(t, s)[H(t, s)]^{1 / 2} \text { for all }(t, s) \in D .
$$

Finally, assume that

$$
\limsup _{t \rightarrow \infty} \frac{1}{H\left(t, t_{0}\right)} \lambda_{1}\left[\int_{t_{0}}^{t}\left(H(t, s) Q(s)-\frac{1}{4} h^{2}(t, s) P(s)\right) d s\right]=\infty .
$$

Then system (1.1) is oscillatory.

Proof. Suppose to the contrary that there exists a prepared solution $Y(t)$ of (1.1) which is not oscillatory. Without loss of generality, we may suppose that $\operatorname{det} Y(t) \neq 0$ for $t \geq t_{0}$. Define $V(t)=P(t) Y^{\prime}(t) Y^{-1}(t)$. We obtain the Riccati equation

$$
Q(t)=-V^{\prime}(t)-V(t) P^{-1}(t) V(t), \quad t \geq t_{0} .
$$

Multiplying (2.2), with $t$ replaced by $s$, by $H(t, s)$ and integrating from $t_{0}$ to $t$, we obtain

$$
\begin{aligned}
\int_{t_{0}}^{t} H(t, s) Q(s) d s=-\int_{t_{0}}^{t} H(t, s) V^{\prime}(s) d s-\int_{t_{0}}^{t} H(t, s) V(s) P^{-1}(s) V(s) d s \\
=H\left(t, t_{0}\right) V\left(t_{0}\right)-\int_{t_{0}}^{t}\left[-H_{s}(t, s)\right] V(s) d s-\int_{t_{0}}^{t} H(t, s) V(s) P^{-1}(s) V(s) d s \\
=H\left(t, t_{0}\right) V\left(t_{0}\right)-\int_{t_{0}}^{t} h(t, s)[H(t, s)]^{1 / 2} V(s) d s \\
\quad-\int_{t_{0}}^{t} H(t, s) V(s) P^{-1}(s) V(s) d s .
\end{aligned}
$$

Since $P(t)>0$, let $R(t)=\left[P^{-1}(t)\right]^{1 / 2}$. We have

$$
\begin{aligned}
& \int_{t_{0}}^{t} H(t, s) Q(s) d s \\
&=H\left(t, t_{0}\right) V\left(t_{0}\right)-\int_{t_{0}}^{t} h(t, s)[H(t, s)]^{1 / 2} R^{-1}(s)[R(s) V(s) R(s)] R^{-1}(s) d s \\
&-\int_{t_{0}}^{t} H(t, s) R^{-1}(s)[R(s) V(s) R(s)][R(s) V(s) R(s)] R^{-1}(s) d s \\
&= H\left(t, t_{0}\right) V\left(t_{0}\right)+\frac{1}{4} \int_{t_{0}}^{t} h^{2}(t, s) P(s) d s \\
&-\int_{t_{0}}^{t} R^{-1}(s)\left\{[H(t, s)]^{1 / 2}[R(s) V(s) R(S)]+\frac{1}{2} h(t, s) I\right\}^{2} R^{-1}(s) d s .
\end{aligned}
$$

Hence we have

$$
\int_{t_{0}}^{t}\left(H(t, s) Q(s)-\frac{1}{4} h^{2}(t, s) P(s)\right) d s \leq H\left(t, t_{0}\right) V\left(t_{0}\right), \quad t \geq t_{0} .
$$

It follows that

$$
\lambda_{1}\left[\int_{t_{0}}^{t}\left(H(t, s) Q(s)-\frac{1}{4} h^{2}(t, s) P(s)\right) d s\right] \leq \lambda_{1}\left[H\left(t, t_{0}\right) V\left(t_{0}\right)\right] .
$$


Since $h\left(t, t_{0}\right)>0$ for $t>s \geq t_{0}$, dividing (2.4) by $H\left(t, t_{0}\right)$ we get

$$
\begin{gathered}
\frac{1}{H\left(t, t_{0}\right)} \lambda_{1}\left[\int_{t_{0}}^{t}\left(H(t, s) Q(s)-\frac{1}{4} h^{2}(t, s) P(s)\right) d s\right] \\
\leq \frac{1}{H\left(t, t_{0}\right)} \lambda_{1}\left[H\left(t, t_{0}\right) V\left(t_{0}\right)\right]=\lambda_{1}\left[V\left(t_{0}\right)\right] .
\end{gathered}
$$

Taking the upper limit in both sides of (2.5) as $t \rightarrow \infty$, the right-hand side is always bounded, which contradicts condition (2.1). This completes the proof.

Under a modification of the hypotheses of Theorem 1, we can obtain the following result.

Theorem 2. In Theorem 1, if condition (2.1) is replaced by the conditions

$$
\limsup _{t \rightarrow \infty} \frac{1}{H\left(t, t_{0}\right)} \lambda_{1}\left[\int_{t_{0}}^{t} h^{2}(t, s) P(s) d s\right]<\infty
$$

and

$$
\limsup _{t \rightarrow \infty} \frac{1}{H\left(t, t_{0}\right)} \lambda_{1}\left[\int_{t_{0}}^{t} H(t, s) Q(s) d s\right]=\infty,
$$

then system (1.1) is oscillatory.

Let $P(t)=\operatorname{diag}\left(p_{1}(t), p_{2}(t), \ldots, p_{n}(t)\right)$ where $p_{i}(t)$ is continuous and positive for $t \geq t_{0}, i=1,2, \ldots, n$. Let $p(t)=\max _{1 \leq i \leq n}\left\{p_{i}(t)\right\}$.

Theorem 3. In Theorem 2, if condition (2.6) is replaced by

$$
\limsup _{t \rightarrow \infty} \frac{1}{H\left(t, t_{0}\right)} \int_{t_{0}}^{t} h^{2}(t, s) p(s) d s<\infty,
$$

then system (1.1) is oscillatory provided (2.7) holds.

If $P(t) \equiv I$ for $t \geq t_{0}$, we have the following result.

Theorem 4. Let $H$ and $h$ be as in Theorem 1. Assume that

$$
\limsup _{t \rightarrow \infty} \frac{1}{H\left(t, t_{0}\right)} \int_{t_{0}}^{t} h^{2}(t, s) d s<\infty
$$

and (2.7) holds. Then system (1.2) is oscillatory.

Choosing different functions $H(t, s)$ and $h(t, s)$ in Theorems $1,2,3$, and 4 , we can obtain various oscillation criteria for systems (1.1) and (1.2).

First, let us consider the function $H$ defined by

$$
H(t, s)=(t-s)^{m-1}, \quad t \geq s \geq t_{0},
$$

where $m$ is an integer with $m>2$. Then $H(t, t)=0, H(t, s)>0$ for $t>s \geq t_{0}$, and $H_{s}(t, s)=-(m-1)(t-s)^{m-2}$ is nonpositive and continuous for $t \geq s \geq t_{0}$. Then

$$
h(t, s)=(m-1)(t-s)^{(m-3) / 2}, \quad t \geq s \geq t_{0} .
$$

Hence, by Theorem 4, we obtain 
Theorem 5. Let $m>2$ be an integer. Assume that

$$
\limsup _{t \rightarrow \infty} \frac{1}{t^{m-1}} \lambda_{1}\left[\int_{t_{0}}^{t}(t-s)^{m-1} Q(s) d s\right]=\infty .
$$

Then system (1.2) is oscillatory.

Remark 1. If $Q(t) \equiv q(t)$, a scalar function, then system (1.2) becomes the scalar equation (1.4), and the oscillation criterion (2.11) becomes the Kamenev criterion (1.8). It is well known that the weaker condition

$$
\limsup _{t \rightarrow \infty} \int_{t_{0}}^{t} q(s) d s=\infty
$$

is not a sufficient condition for oscillation of the scalar equation (1.4), and consequently, the condition (cf. [5])

$$
\limsup _{t \rightarrow \infty} \lambda_{1}\left[\int_{t_{0}}^{t} Q(s) d s\right]=\infty
$$

is not a sufficient condition for oscillation of the matrix system (1.2). Our Theorem 5 extends the Kamenev type criterion (1.8) to the matrix system (1.2).

Next, consider the function

$$
H(t, s)=\left[\ln \frac{t}{s}\right]^{m-1}, \quad t \geq s \geq t_{0}
$$

Then

$$
h(t, s)=\frac{m-1}{s}\left[\ln \frac{t}{s}\right]^{(m-3) / 2}, \quad t \geq s \geq t_{0} .
$$

By Theorem 4, we have

Theorem 6. Let $m>2$ be an integer. Assume that

$$
\limsup _{t \rightarrow \infty} \frac{1}{(\ln t)^{m-1}} \lambda_{1}\left[\int_{t_{0}}^{t}\left(\ln \frac{t}{s}\right)^{m-1} Q(s) d s\right]=\infty .
$$

Then system (1.2) is oscillatory.

Now, let $H(t, s) \equiv \rho(t-s)$ where $\rho(u)$ is a continuously differentiable function on $[0, \infty), \rho(0)=0, \rho(u)>0, \rho^{\prime}(u) \geq 0$ for $u>0$. Then

$$
h(t, s)=-\frac{\rho^{\prime}(t-s)}{\rho^{1 / 2}(t-s)}, \quad t \geq s \geq 0 .
$$

By Theorem 4 we have the following results.

Theorem 7. Assume that there exists a continuously differentiable function $\rho(u)$ on $[0, \infty), \rho(0)=0, \rho(u)>0$, and $\rho^{\prime}(u) \geq 0$ for $u>0$ such that

$$
\limsup _{t \rightarrow \infty} \frac{1}{\rho(t)} \int_{0}^{t} \frac{\left[\rho^{\prime}(t-s)\right]^{2}}{\rho(t-s)} d s<\infty
$$

and

$$
\limsup _{t \rightarrow \infty} \frac{1}{\rho(t)} \lambda_{1}\left[\int_{0}^{t} \rho(t-s) Q(s) d s\right]=\infty .
$$

Then system (1.2) is oscillatory. 
Remark 2. Theorem 7 extends Corollary 9 of Kwong and Kaper [13], where system (1.2) with $n=2$ is considered. By Theorem 1, we can also establish a similar result which extends Theorem 8 of [13].

Remark 3. More general Kamenev type criteria in Philos [16] and Yan [20] can be extended to matrix system (1.2) similarly.

\section{REFERENCES}

1. W. Allegretto and L. Erbe, Oscillation criteria for matrix differential inequalities, Canad. Math. Bull. 16 (1973), 5-10.

2. F. V. Atkinson, H. G. Kaper, and M. K. Kwong, An oscillation criterion for linear secondorder differential systems, Proc. Amer. Math. Soc. 94 (1985), 91-96.

3. G. J. Butler and L. H. Erbe, Oscillation results for second order differential systems, SIAM J. Math. Anal. 17 (1986), 19-29.

4. __ Oscillation results for self-adjoint differential systems, J. Math. Anal. Appl. 115 (1986), 470-481.

5. G. J. Butler, L. H. Erbe, and A. B. Mingarelli, Riccati techniques and variational principles in oscillation theory for linear systems, Trans. Amer. Math. Soc. 303 (1987), 263-282.

6. R. Byers, B. J. Harris, and M. K. Kwong, Weighted means and oscillation conditions for second order matrix differential equations, J. Differential Equations 61 (1986), 164-177.

7. G. J. Etgen and R. T. Lewis, Positive functionals and oscillation criteria for second order differential systems, Proc. Edinburgh Math. Soc. (2) 22 (1979), 277-290.

8. B. Fite, Concerning the zeros of the solutions of certain differential equations, Trans. Amer. Math. Soc. 19 (1918), 341-352.

9. P. Hartman, Ordinary differential equations, Wiley, New York, 1982.

10. __ Oscillation criteria for self-adjoint second order differential systems and "principal sectional curvatures", J. Differential Equations 34 (1979), 326-338.

11. D. B. Hinton and R. T. Lewis, Oscillation theory of generalized second order differential equations, Rocky Mountain J. Math. 10 (1980), 751-761.

12. I. V. Kamenev, Integral criterion for oscillations of linear differential equations of second order, Mat. Zametki 23 (1978), 249-251.

13. M. K. Kwong and H. G. Kaper, Oscillation of two-dimensional linear second order differential systems, J. Differential Equations 56 (1985), 195-205.

14. M. K. Kwong, H. G. Kaper, K. Akiyama, and A. B. Mingarelli, Oscillation of second order differential systems, Proc. Amer. Math. Soc. 91 (1984), 85-91.

15. A. B. Mingarelli, On a conjecture for oscillation of second order differential systems, Proc. Amer. Math. Soc. 82 (1981), 593-598.

16. Ch. G. Philos, Oscillation theorems for linear differential equations of second order, Arch. Math. (Basel) 53 (1989), 482-492.

17. E. E. Tomastik, Oscillation of systems of second order differential equations, J. Differential Equations 9 (1971), 436-442.

18. T. Walters, $A$ characterization of positive linear functionals and oscillation criteria for matrix differential equations, Proc. Amer. Math. Soc. 78 (1980), 198-202.

19. A. Wintner, A criterion of oscillatory stability, Quart. Appl. Math. 7 (1949), 115-117.

20. J. Yan, Oscillation theorems for second order linear differential equations with damping, Proc. Amer. Math. Soc. 98 (1986), 276-282.

Department of Mathematics, University of Alberta, Edmonton, Alberta, Canada T6G 2G1 